\title{
Manipulations programmées dans le texte littéraire numérique pour la jeunesse: gestes et actions de compréhension et d'interprétation
}

\section{Screen manipulations in children's digital fictional texts: Comprehension and interpretation gestures and actions}

\author{
Eleonora Acerra \\ Université du Québec en Abitibi-Témiscamingue, Rouyn-Noranda (Québec, Canada)
}

\begin{abstract}
Résumé. En tant que textes interactifs et ergodiques, les œuvres littéraires numériques contemporaines, francophones et non, programment l'intervention empirique de l'interlecteur rice sur l'écran comme une condition d'actualisation du texte et du sens. Le corps de l'interlecteur-rice et son « effort non trivial» (Aarseth, 1997) sont nécessaires pour la traversée de l'œuvre et pour le dévoilement de ses contenus, qui se déclenchent justement grâce à ses gestes et actions. Loin d'être anodines ou purement mécaniques, ces manipulations de l'écran et de l'espace fictionnel requièrent un effort interprétatif, qui les rend actualisables et signifiantes. Cependant, à ce jour, peu d'études se sont intéressées au rôle précis des gestes et des actions des interlecteur'rice's en tant que manifestation d'un parcours interprétatif. Dans cet article, nous faisons état d'une expérience de terrain, menée avec un groupe d'enfants d'école primaire, que nous avons confronté·e·s à un échantillon d'œuvres littéraires numériques conçues pour écran tactile. Nous souhaitions observer leur compréhension, leur interprétation et leur manière de répondre gestuellement à la programmation de l'œuvre.
\end{abstract}

\begin{abstract}
As interactive and ergodic texts, contemporary digital literary creations program the inter-reader's intervention on the screen as a condition for actualizing their text and meaning. The inter-readers' bodies and their "non-trivial effort" (Aarseth, 1997) are necessary for traversing the works and unveiling the contents, which are precisely revealed thanks to their gestures and actions. Far from being mechanical, these screen and narrative manipulations require an interpretive effort, which makes them actualizable and meaningful. However, to these days, only few studies have focused on the precise role of the inter-readers' gestures and actions as a manifestation of an interpretive path. In this article, we discuss a field experience, conducted with a group of young readers discovering a sample of digital literary works designed for touch screens. We will consider on one side how they understand and interpret the story, on the other side, how they respond with gestures and actions to the digital creations expectations.
\end{abstract}




\section{Introduction}

L'introduction des dispositifs tactiles et mobiles dans le champ des pratiques littéraires numériques a provoqué, au-delà de la stimulation d'un segment éditorial resté jusqu'à ce jour assez confidentiel ${ }^{1}$, l'irruption prépondérante de la dimension gestuelle, qui s'est trouvée mise au cœur de la dynamique de l'œuvre. Dans la création littéraire numérique contemporaine pour supports tactiles, gestes et actions ${ }^{2}$ représentent en effet une condition nécessaire pour l'actualisation du texte et pour le déploiement des contenus, le répertoire des mouvements possibles étant bien "plus étendu qu'avec le seul input souris-clavier » (Bouchardon, 2011). Délivrés des contraintes technologiques et matérielles dues à la médiation des dispositifs de pointage, les gestes et les actions peuvent consister, en fonction des œuvres et des circonstances narratives, à tapoter plus ou moins vigoureusement sur l'écran, à gratter, tirer, souffler, dérouler, scroller, relâcher et déplacer des éléments, ou encore à incliner le dispositif, à écrire, photographier ou dessiner. Généralement liés au contexte de lecture et en partie issus de références et analogies avec leurs homologues dans le monde réel, les gestes et les actions programmés par les œuvres numériques explorent les manipulations possibles sur l'écran et sur l'interface narrative, en conférant au corps des interlecteur·rice's (Weissberg, 1999) une double fonction, à la fois textuelle et interprétative. En tant qu'unité textuelle, le corps des interlecteur rice's se voit inscrit dans le code informatique, avec l'ensemble des mouvements empiriques par lesquels il est prévu qu'il se manifeste dans l'espace fictionnel. En même temps, en tant que vecteur d'une réponse empirique aux requêtes d'intervention des œuvres, il est envisagé comme un instrument intellectuel et interprétatif, permettant aux gestes et aux actions programmés de se déployer et d'actualiser le sens que peut prendre l'œuvre. Dès lors, la fonction performative des actions et des gestes permet, d'une part, d'actualiser une signification potentialisée, d'autre part, de réaliser une expérience corporelle qui dépasse le programme narratif envisagé en amont de l'expérience de lecture. Intégré dans l'œuvre numérique en tant qu'élément textuel stratégique et en tant qu'entité empirique, le corps des interlecteur·rice·s s'avère, ainsi, le déclencheur d'une réponse du dispositif ou de l'interface narrative, et la manifestation d'un parcours interprétatif singulier, chaque geste et action découlant d'une réponse subjective aux attentes de l'œuvre.

Le corpus littéraire numérique pour la jeunesse partage avec le répertoire d'œuvres destinées à d'autres publics la même centralité de la dimension gestuelle. En fonction des œuvres et des contextes, les jeunes interlecteur·rice·s sont censé·e·s faire de leurs gestes et actions une partie intégrante du tissu littéraire, en intervenant avec leurs doigts pour tracer le passage dans l'œuvre, dévoiler et manier les matières textuelles, activer des fonctionnalités ou, encore, pour sélectionner un parcours narratif au sein d'un réseau de chemins possibles. Dans ce cas aussi, chaque geste et action peut être considéré comme la manifestation d'une démarche interprétative, voire comme une forme d' ' interprétation actualisée » (Jeanneret, 2000), s'explicitant par un mouvement - ou une concaténation de mouvements - volontaires et contextualisés sur l'écran. En agissant leur interprétation, les jeunes interlecteur·rice·s sont appelé·e·s à réaliser les actions et les gestes qui leur semblent cohérents avec les situations proposées et, en même temps, se doivent d'accorder leurs mouvements à la programmation de l'œuvre, qui a déterminé en amont les finalités de l'intervention et les possibilités d'actualisation (Eco, 1985). Cette configuration présuppose à la fois la mobilisation de compétences et d'habilités spécifiques, liées aux dimensions technologique et littéraire du texte numérique, et la volonté d'adhérer à cette utilisation du corps à des fins narratives.

Se conformer au parcours stratégiquement et informatiquement établi par l'œuvre s'avère d'autant plus complexe que la prétendue liberté d'interaction promue par les interfaces tactiles est de facto limitée par la configuration technolittéraire de l'œuvre numérique: en effet, d'une part, les œuvres littéraires numériques, comme tout produit littéraire, n'admettent qu'un nombre limité d'interprétations et donc d'actualisations; d'autre part, le code informatique conditionne les mouvements interprétatifs possibles, en fixant un réseau restreint de solutions gestuelles acceptables au vu du contexte et du dispositif. Dans cette perspective, qui contraint le "pouvoir d'action » (Murray, 1997) des interlecteur·rice's aussi bien au vu des affordances des «modes sémiotiques » et des choix des « concepteur·rice·s " (Bezemer et Kress, 2015 : 133) qu'à la nature même du texte numérique, la manière d'actualiser un parcours sur écran, de déclencher l'avancement narratif, de dévoiler ou d'animer les contenus peut être considérée comme un indicateur de la posture interprétative du sujet qui lit. Selon cette hypothèse, gestes et actions traduisent et matérialisent le processus de compréhension-interprétation de la scène et des manipulations perçues comme étant attendues, en rendant également visible la manière dont les interlecteur 'rice's choisissent d'endosser le rôle d'instrument de la narration que l'œuvre leur a conféré.

Cette réponse corporelle à la programmation de l'œuvre permet de penser l'intervention sur l'écran comme une forme d'expression d'agentivité. L'agentivité, c'est ici ce qui permet à l'interlecteur'rice de jouer un rôle dans le développement de sa compétence à lire et de s'adapter au fonctionnement du dispositif de lecture et au dispositif de

\footnotetext{
${ }^{1}$ Selon Bélisle (2011), le moment décisif pour le secteur éditorial numérique a cö̈ncidé avec l'arrivée sur le marché de l'iPad. Sa diffusion massive et extrêmement rapide, en 2010, a « eu un réel effet de stimulation » (p. 189) et mené « les éditeurs et les grands groupes de presse (...) à développer des offres spécifiques pour transposer leur production de livres papier sur des tablettes numériques» (p. 189). En parallèle, de nouvelles œuvres, développées aussi bien par des studios de développement, des éditeur·rice's pure players (soit œuvrant uniquement dans le milieu numérique) et des acteur·rice's de l'industrie audiovisuelle, ont vu le jour.

${ }^{2}$ Nous nous appuyons sur la distinction entre gestes et actions posée par Bouchardon (2011). Le chercheur définit le geste comme une « activité unitaire (appui sur une touche du clavier ou sur le bouton de la souris, déplacement élémentaire de la souris, etc.) » et l'action « un énoncé de gestes (par exemple le glisser/déposer - drag and drop)» (Bouchardon, 2011 : 39).
} 
l'œuvre. Impliquant un principe d'autodétermination et d'autorégulation, ainsi qu'un «sentiment affirmé de son efficacité personnelle à apprendre » (Carré, 2003), elle peut être définie comme la capacité à agir de manière intentionnelle dans l'environnement dans lequel l'on se trouve (Bandura, 2006). Issue de facteurs cognitifs, socioaffectifs et épistémiques individuels, dans la lecture d'œuvres fictionnelles numériques, elle permet de relier les gestes et les actions réalisés à l'écran à un comportement subjectif et motivé, qui traduit l'exercice interprétatif du sujet et son désir de voir le texte actualisé.

Cette contribution se propose d'interroger le rapport corporel à l'interprétation, en observant plus particulièrement l'ensemble des mouvements empiriques et interprétatifs effectués par un échantillon de jeunes interlecteur·rice $\cdot s$ lors de la découverte d'un corpus d'applications et d'ePubs littéraires pour la jeunesse. Nous chercherons à vérifier si les gestes et les actions effectués lors de la lecture d'œuvres conçues pour des écrans tactiles et mobiles traduisent une forme d'agentivité ou bien s'ils sont le produit d'une réponse mécanique aux requêtes d'interaction du dispositif. Ce faisant, nous questionnerons également la perception que les interlecteur'rice's ont de leur propre rôle dans l'environnement littéraire numérique et leur manière de se conformer à cette double programmation, informatique et littéraire, du texte numérique. Après une introduction permettant d'ancrer notre recherche à la réflexion théorique sur la rhétorique de la réception et sur les gestes de manipulation, nous présenterons les résultats d'une observation de terrain, menée avec des élèves d'une école primaire à Montréal (Québec).

\section{Gestes et actions comme " interprétations actualisées ${ }^{3}$ »}

Le «tournant tactile» (Pelard, 2018) de la création littéraire numérique a resserré ces liens entre corps, écran et multitexte qui ont toujours caractérisé les pratiques de littérature numérique. Tant les clics dans des blocs textuels des premiers hypertextes fictionnels que les manipulations prévoyant des associations entre signes linguistiques et iconiques dans les œuvres plus récentes se fondent sur la dimension signifiante du " geste », qui « révèle la matérialité du texte » (Bouchardon, 2009) et permet, en même temps, de construire le sens. Dans un texte numérique, déclencher une animation, effleurer un flux de mots, explorer un espace tridimensionnel ou, encore, agir sur des objets à l'écran constituent autant de manières pour dévoiler des couches multitextuelles autrement invisibles, ainsi que pour les rendre signifiantes. Dans Déprise (Bouchardon, 2010), par exemple, le mouvement des interlecteur rice's met au jour le sentiment de perte de contrôle éprouvé par le protagoniste du récit. Ses impulsions provoquent l'éclatement du texte, qui s'envole et s'émiette quand on cherche à l'appréhender ; en même temps, elles déclenchent l'arrivée d'une série de bulles colorées, qui s'éparpillent aléatoirement sur l'écran, comme autant de particules flottant dans un espace régi par des lois externes et inéluctables (Figures 1a et 1b). Le corps des interlecteur·rice·s, moyen d'expression de l'agentivité, détermine l'apparition des contenus et devient l'instrument permettant à la métaphore de la fragilité et de la dérive qui domine l'œuvre de se matérialiser.
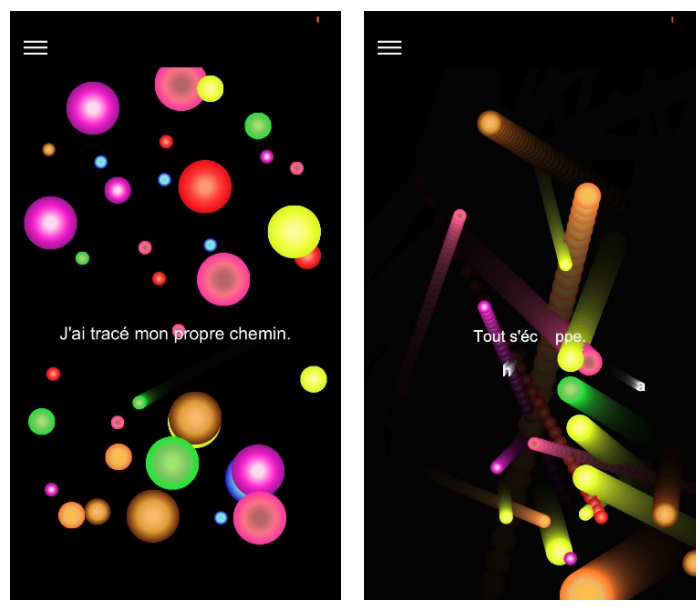

Figures 1a et 1b. Déprise (Bouchardon, 2010).

Pour que cette intervention soit réalisée et signifiante, l'interlecteur rice se doit d'associer le « signe lu», l'« outil manipulable » et le « signe interprété » (Jeanneret et Souchier, 1999). Cela équivaut à réagir aux «promesses de sens » (Saemmer, 2007) portées par l'ensemble de «signes passeurs » (Jeanneret, $2000: 113$ ) auxquels il·elle est confronté·e et à opérer des choix critiques, issus d'une analyse sémiotique et métacognitive. Les hyperliens, tout comme les autres éléments iconotextuels avec lesquels on peut interagir dans le texte numérique, portent en eux «un ordre, une suggestion d'aller plus loin. Lorsque l'interlecteur·rice clique, il·elle obéit à l'ordre, à la permission ou à la suggestion »

${ }^{3}$ Jeanneret, $2000: 170$. 
(Saemmer, 2007 : 58). En même temps, il·elle accepte de s'aventurer et de naviguer dans l'espace virtuel, en suivant son gout pour la découverte, ainsi que ses intuitions par rapport aux contextes et aux mouvements attendus. Dès lors, comme rappelé par Yves Jeanneret, si « le fait de tourner la page ne suppose a priori aucune interprétation particulière du texte, dans le multimédia, le fait de cliquer sur un mot (un « hypermot») ou sur un pictogramme (une « icône ») est, en lui-même un acte d'interprétation» (2000: 112-113). Il comporte un positionnement de l'interlecteur'rice et un investissement, intellectuel et physique, dans le texte et avec l'écran.

Dans l'œuvre pour la jeunesse, tout comme dans la production pour adultes, gestes et actions constituent la réponse aux mouvements programmés par l'œuvre et les outils par lesquels elle se déploie. Ils découlent aussi d'une compréhensioninterprétation de la scène et des attentes - ou «préfigurations » (Saemmer, 2015) - de l'œuvre envers son destinataire. Les travaux d'Alexandra Saemmer ont permis de conceptualiser ce rapport entre attentes et promesses de sens, ainsi qu'entre medium et message, en pensant l'œuvre numérique au prisme d'une " rhétorique de la réception » (Saemmer, 2015), qui prend en compte le texte programmé, avec l'ensemble de ses "procédés de disposition logique et ses matérialités », et un « horizon d'attente extratextuel » (Saemmer et Tréhondart, $2014: 109)$, concernant les destinataires et composé à partir de leurs " imaginaires, attentes, espoirs et habitudes » de lecture (Saemmer et Tréhondart, 2014 : 109). Selon cette perspective, le texte numérique véhicule, avec son message, son horizon de réception, qu'il «préfigure (...) à travers ses contenus et ses procédés rhétoriques" (Saemmer, 2015: 14), ainsi qu'à travers "ses formes graphiques sur la page-écran » (Saemmer, 2015: 13). Reconnaissables par voie "d'intégration et de stabilisation d'expériences antérieures » (Saemmer, $2015: 48$ ), mais également par rapport à un potentiel d' " irradiation iconique » (Saemmer, 2015 : 49) susceptible de varier en fonction de la familiarité avec les dispositifs ou de la prévisibilité de l'association engagée par le couplage médiatique, sémiotique ou gestuel, les dispositions par lesquelles le texte numérique se donne à lire, à voir et à manipuler contribuent à l'expression de son esthétique et conditionnent les moyens de la réception. Gestes et actions témoignent ainsi d'un rapport subjectif entre l'interlecteur-rice et le texte (fictionnel et non) et, en même temps, de sa capacité à reconnaitre d'éventuels rapprochements ou analogies avec des référents d'expérience: dans l'œuvre numérique, on presse pour afficher des menus ou pour révéler des éléments cachés, on glisse pour déplacer des objets, on tapote sur des zones d'intérêt, on pince pour zoomer ou on élargit pour dé-zoomer, on souffle pour faire disparaitre un texte ou une image (Saemmer et Tréhondart, 2014). Par cela, le corps des interlecteur·rice's seconde l'expérience sensorielle à laquelle il·elle est invité·e et, en même temps, se prête à investir le monde de l'écran d'une connaissance du monde réel.

\subsection{Gestes et actions dans une « rhétorique de la manipulation ${ }^{4}$ »}

La gestualisation de la lecture (Jeanneret, 2000) est strictement liée à trois caractéristiques du texte numérique : l'ergodisme, l'interactivité et la prégnance de la dimension matérielle. Par le premier terme, introduit en 1997 par Espen Aarseth, on définit toute œuvre, numérique comme analogique, requérant un « effort non trivial » (Aarseth, 1997) pour être traversée, lue, comprise et révélée. Plus significatives que des actions réalisées mécaniquement en feuilletant les pages d'un livre, ou en laissant courir le regard d'une ligne à l'autre, les manipulations présupposées par une production ergodique n'intéressent pas uniquement le dispositif ou le support, mais également le « texte lui-même » (Bouchardon, 2011). Ce dernier, de par son caractère programmé et son interactivité, est en mesure de répondre aux impulsions du destinataire : «le support réagit et exécute certaines actions» (Bouchardon, 2014), en permettant ainsi aux interlecteur·rice's de naviguer à travers l'œuvre, de la manipuler ou, éventuellement, de l'enrichir de nouvelles données. Le caractère programmé de l'œuvre et sa capacité à faire correspondre à chaque mouvement de l'interlecteur·rice un changement sur l'écran révèlent aussi la matérialité de l'écrit numérique (Bouchardon, 2008b). Ils donnent à voir le «processus matériel » interne à la machine et « ce qui se passe dans l'interaction avec l'utilisateur rice » (Bouchardon, 2008a). Dès lors, ils permettent de combiner les propriétés physiques du dispositif et les ressources iconotextuelles ou sonores pour tisser les contenus et faire jaillir des effets de sens, voire des « figures » stylistiques et rhétoriques au sens propre. Plus particulièrement, selon les chercheur $\cdot e \cdot s$ en littérature numérique, dans le texte numérique, on peut distinguer, "à côté des figures de diction, de construction, de sens (ou tropes) et de pensée » (Bouchardon, 2008a) issues de la rhétorique ou de la stylistique classiques, des "phénomènes de sens émergeant de la combinaison entre le mouvement, la manipulation et le texte ou l'image » (Saemmer, $2011: 23$ ). Les travaux d'Alexandra Saemmer et de Serge Bouchardon ont notamment permis d'identifier des figures d'animation et de manipulation, issues de « couplages" plus ou moins «conventionnels» entre ressources iconotextuelles, sonores et gestes lectoraux. Des figures d'apparition/disparition, de substitution et de déplacement (Bouchardon, 2008a), des métonymies et métalepses interactives (Saemmer, 2008), ainsi que des kiné-grammes et des ciné-grammes ont été également repérées et caractérisées. Dans la fiction pour la jeunesse, des figures de la disposition textuelle, de l'opposition, de la focalisation, de la description, de l'exploration, de la performativité et de la textonique ont été reconnues (Acerra, 2019) : elles permettent de décrire, respectivement, des circonstances narratives dans lesquelles l'interlecteur·rice contribue à organiser la matière sur la page-écran, se fraye un chemin dans un texte qui résiste à son passage, déclenche un changement de perspective, de point de vue ou une description, se laisse transporter dans des activités non directement liées à l'expérience fictionnelle, actualise des actions et des gestes qui coïncident avec les actes qu'ils décrivent ou encore s'attache à modifier de manière permanente la façon dont textes et illustrations s'affichent à l'écran.

${ }^{4}$ Bouchardon, 2008a. 
Malgré la richesse de procédés par lesquels cette «rhétorique de la manipulation » se met en œuvre et bien que le mouvement empirique de l'interlecteur'rice soit crucial pour son fonctionnement, "peu de chercheur.e.s se sont penché·e·s sur le rôle précis du geste dans son interaction avec le texte numérique » (Saemmer, 2015:46). En ce qui concerne les jeunes lecteur·rice·s, tout particulièrement, les recherches ciblant à la fois la finalité de gestes et actions ou la perception de leurs apports pour l'œuvre sont, à ce jour, très rares et ne ciblent pas nécessairement la démarche interprétative qui nous préoccupe.

Pour cette raison, nos travaux ciblent celles que nous proposons d'appeler des manipulations narratives programmées. Par cette définition, nous insistons sur les trois dimensions qui définissent l'interaction dans le corpus littéraire tactile et mobile : la démarche haptique qu'elles présupposent (soulignée par le terme manipulation), l'aspect informatique qui leur permet de se déployer (valorisé par l'adjectif programmée) et le niveau dans lequel l'interaction a lieu ou est susceptible de déterminer un effet (narratif). Assimilables à une propriété intrinsèque du texte numérique et à une condition de son actualisation, ces interventions présupposent une démarche interprétative spécifique, qui justifie et rend signifiants les mouvements attendus.

\section{L'expérimentation}

Selon le postulat développé dans ce travail, en tant que texte codé informatiquement pour être interactif et ergodique, l'œuvre littéraire numérique présuppose à la fois la coopération interprétative d'un destinataire type et la participation d'un'e agent·e empirique, capable de traduire sa lecture, sa compréhension et son interprétation en mouvements réels et délibérés sur l'interface narrative. Dès lors, le corps des interlecteur·rice's peut être considéré à plein titre comme l'une des mailles stratégiques de l'actualisation du texte et de sa recevabilité, car, sans le corps des interlecteur·rice·s, le corps $\mathrm{du}$ texte ne se donne pas. Comment alors les jeunes interlecteur $\cdot$ rice $s$ répondent-il $\cdot e l l e \cdot s$ aux requêtes d'interactions des œuvres numériques et que peut-on dire de leurs "interprétations actualisées »? Leurs gestes et actions sont-ils le produit d'une démarche interprétative consciente ou bien d'une réponse mécanique aux requêtes d'interaction du dispositif? Le corps est-il perçu comme un instrument au service de la narration ? Afin de mettre à l'épreuve nos hypothèses et répondre à ces questions, nous avons choisi d'observer les conduites interprétatives (gestuelles, verbales et paraverbales) d'un groupe de jeunes interlecteur·rice·s, que nous avons confronté·e·s à un échantillon d'œuvres conçues pour support mobile. Nous nous sommes concentrée plus particulièrement sur une sélection d'applications et d'ePubs littéraires, d'une part, car ces formats représentent un segment éditorial en croissance exponentielle, d'autre part, car leurs configurations technologique et littéraire invitent à une expérience de lecture multisensorielle.

\subsection{Méthodologie}

L'expérience, réalisée à l'automne 2016 dans une école élémentaire montréalaise, a impliqué neuf enfants québécois·e·s agé·e·s entre 8 et 9 ans $^{5}$, que nous avons d'abord filmé·e·s lors d'une séance de découverte d'un échantillon d'applications littéraires, puis interrogé·e·s sur des aspects de leurs compréhension, interprétation et perceptions de l'expérience de lecture lors d'entretiens individuels et semi-structurés d'une durée de 15 minutes ${ }^{6}$. Par le suivi des étapes de découverte et de lecture des œuvres, nous cherchions à observer les réactions aux requêtes d'interaction des applications, ainsi que la manière de traduire en actions et gestes interprétatifs les mouvements supposément attendus par l'écran. Par le recueil et l'analyse des données verbales, nous souhaitions vérifier si les mouvements réalisés étaient effectivement perçus comme « significatifs » et « incorporés dans l'histoire » (Murray, 1997), une simple (inter)action ne suffisant pas forcément à démontrer une attitude critique et sensible vis-à-vis du texte. La grille d'entretien utilisée permettait d'interroger les stratégies de navigation adoptées et d'analyser les retours concernant les actions et les gestes effectivement réalisés par rapport au contexte, tandis que les outils de suivi de la dimension haptique, créés en adaptant le modèle d'analyse des gestes de manipulation proposé par Serge Bouchardon $(2011)^{7}$, offraient un cadre favorable au suivi des actualisations des interlecteur·rice·s par rapport à la programmation des œuvres.

\footnotetext{
${ }^{5}$ Pour assurer l'anonymat des participant $\cdot e \cdot s$, nous avons attribué à chaque enfant un numéro. Dans cet article, nous les nommerons Lecteur 1, Lectrice 2, Lectrice 3, Lecteur 4, Lecteur 5, Lecteur 6, Lectrice 7, Lecteur 8, Lectrice 9.

${ }^{6}$ Toutes les rencontres se sont déroulées dans la bibliothèque de l'établissement, afin d'interroger une forme de lecture différente de celle personnelle (ou familiale) comme scolaire. Par ce choix, nous nous proposions également d'investir un espace déchargé des dynamiques de la classe, tant en termes d'exploitation didactique des œuvres que de construction collective du sens.

${ }^{7}$ Selon Serge Bouchardon, il est possible de distinguer «cinq niveaux d'articulation du signe, correspondant à cinq niveaux d'analyse » (2011: 39). Le premier est représenté par le gestème, qui est défini comme le « résultat d'un couplage entre une activité physique et une interface d'entrée » (p. 39). Le deuxième est l'actème : décliné en trois formes selon la finalité et l'effet du processus de manipulation, il est « fabriqué à partir des gestèmes » et se définit comme le « résultat d'un couplage entre le gestème et le processus sur lequel porte la manipulation» (p. 39). L'unité sémiotique de la manipulation, qui est à son tour composée d'actèmes, se définit avant tout par sa dimension iconique : ses « traits d'iconicité permettent d'insister sur la co-typie, à savoir le lien avec les expériences dans le monde physique» (p. 43). Le couplage média, correspondant au quatrième niveau d'analyse, est défini comme «le geste interfacique qui résulte du couplage entre l'unité sémiotique de manipulation et l'état média environnant » (p. 39) : par "l'intersection» avec les gestes de manipulation, le couplage média permet d'actualiser les traits signifiants des ressources médiatiques ciblées par l'action. Le cinquième niveau d'analyse s'intéresse à la dimension discursive et "prend en
} 
Afin d'observer les comportements des participant·e·s en fonction des spécificités technologiques de chaque texte et des types d'interactions programmés, nous avons sélectionné un corpus de quatre œuvres, programmant un répertoire gestuel suffisamment ample et diversifié pour observer des conduites de manipulation et d'interprétation différentes ${ }^{8}$.

La première histoire, Avec quelques briques (Dieudonne, 2014), est une adaptation en format applicatif d'un album popup de l'artiste Vincent Godeau et relate l'histoire d'un jeune garçon découvrant ses émotions à travers un voyage métaphorique dans son cœur. Dans la version numérique, les mécanismes pop-up de l'œuvre originale ont été transposés en interactions contraignantes pour l'avancement narratif : l'application prévoit, par exemple, de nourrir le jeune garçon en dessinant sa nourriture de briques (Figure 2), ou de provoquer ses larmes en inclinant le dispositif ou en appuyant fortement sur l'écran.

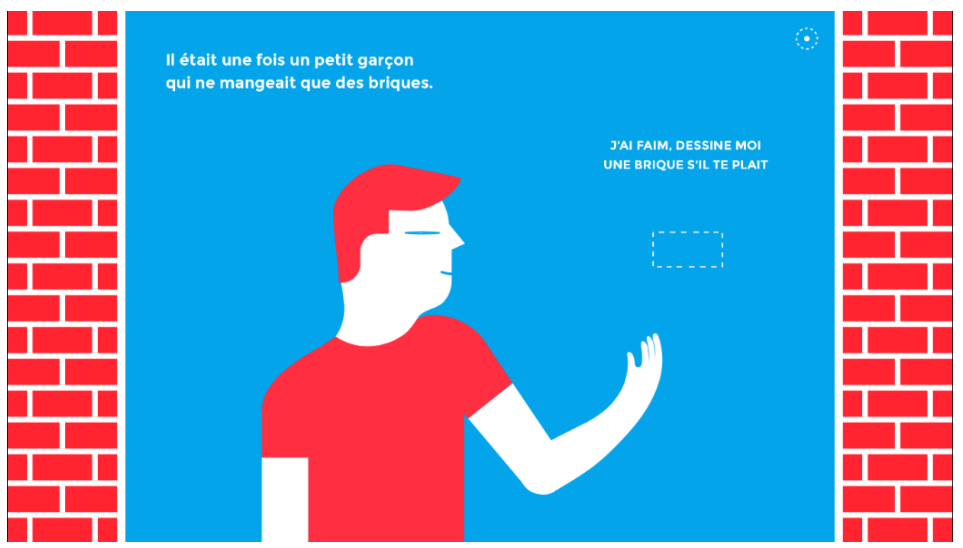

Figure 2. Avec quelques briques (Dieudonne, 2014).

La deuxième œuvre utilisée dans le cadre de cette enquête, Moi, j'attends..., est aussi une adaptation, réalisée par France Télévision (2013), à partir de l'album éponyme de Serge Bloch et David Cali. L'histoire, parcourant la vie d'un personnage, de l'enfance à l'âge mûr, se développe autour de l'image d'un brin rouge, symbolisant le fil de la vie, qui traverse chaque page de l'album. Dans la version numérique, l'interlecteur·rice est censé·e interagir constamment avec le fil, qui change de forme en fonction de la scène : il représente, par exemple, à la fois un cordon ombilical, une couronne funéraire et une querelle entre le protagoniste et son épouse (Figure 3). L'interaction implique donc de comprendre la fonction du fil dans chaque scène et de réagir de manière cohérente aux contextes.

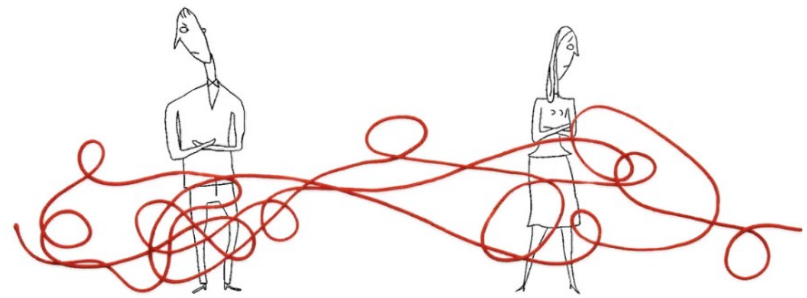

compte l'ensemble du discours interactif» (p. 40) dans lequel le geste de manipulation se déploie : il « correspond à une séquence interactive complète de couplages média » (Bouchardon, 2011:82) et porte donc sur l'ensemble de l'œuvre.

${ }^{8}$ Cette démarche comporte deux biais. Le premier concerne l'observation de la manipulation sur écran, dont on n'observe qu'une manifestation épidermique : n'ayant pas à disposition des outils de suivi oculaire, ni d'outils mécaniques d'enregistrement des gestes réalisés, notre description ne peut pas aspirer à décrire finement l'ensemble des traces gestuelles produites par les lecteur'rice's. En même temps, en interrogeant les conduites verbales, le parcours cognitif et intellectuel dont gestes et actions sont issus demeure en grande partie inaccessible. Le deuxième biais concerne notre propre posture de lectrice : chaque geste, action ou parcours de circulation ont été en effet considérés au prisme d'une appropriation personnelle des œuvres et impliquent donc un regard socialement situé sur les mouvements attendus par les destinataires. 
Figure 3. Moi, j'attends... (France Télévision, 2013).

La troisième adaptation, Mon voisin (Dorléans, 2013), a été choisie car elle propose une manière non conventionnelle de tourner les pages, en combinant un feuilletage vertical et horizontal qui est censé rappeler l'enquête menée par le personnage principal à l'intérieur du bâtiment où se déroule l'histoire. Dans ce cas, pour avancer dans l'histoire, les interlecteur·rice's doivent cliquer soit sur l'une des deux flèches, à travers lesquelles il·elle·s peuvent monter et descendre les étages, soit sur une cloison séparant l'appartement du personnage principal de celui de son voisin (Figure 4).

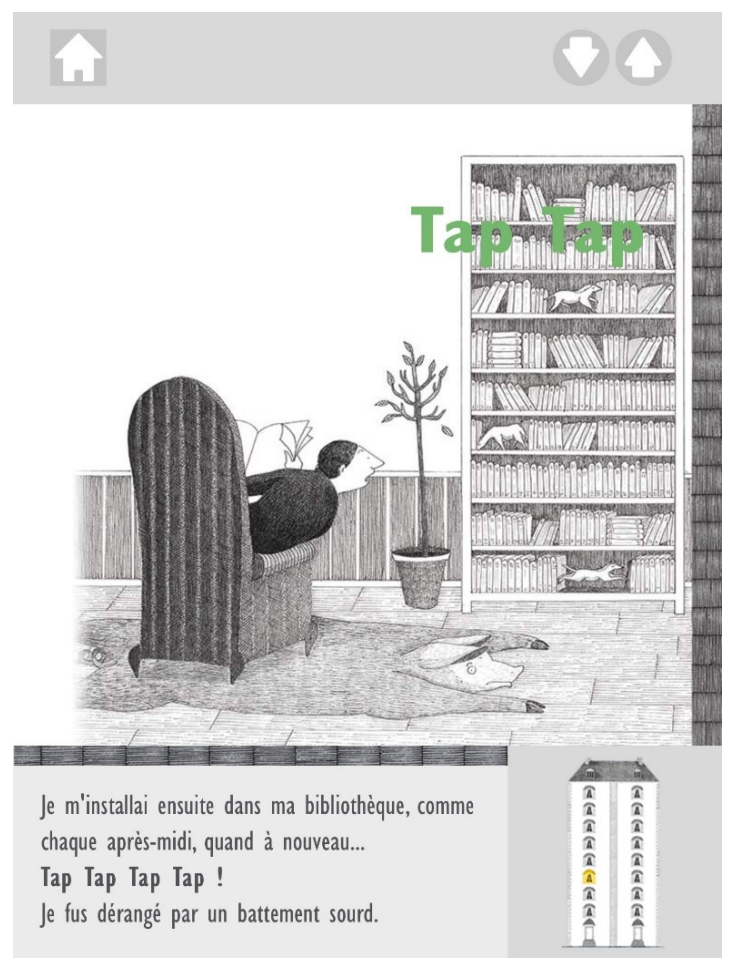

Figure 4. Mon voisin (Éditions des Braques et Tralalere, 2013).

La quatrième création, Le lapin bricoleur (E-toiles, 2015) était la seule œuvre littéraire de notre échantillon non issue de la remédiation d'une source précédente. Choisie en tant que représentative d'un mode de lecture non linéaire, elle propose aux interlecteur $\cdot$ rice's de se frayer leur chemin en sélectionnant parmi différentes possibilités narratives : dès le premier écran, le texte présente une situation et deux options de parcours narratif, dont le choix sera déterminant pour l'issue de l'histoire (Figure 5). 


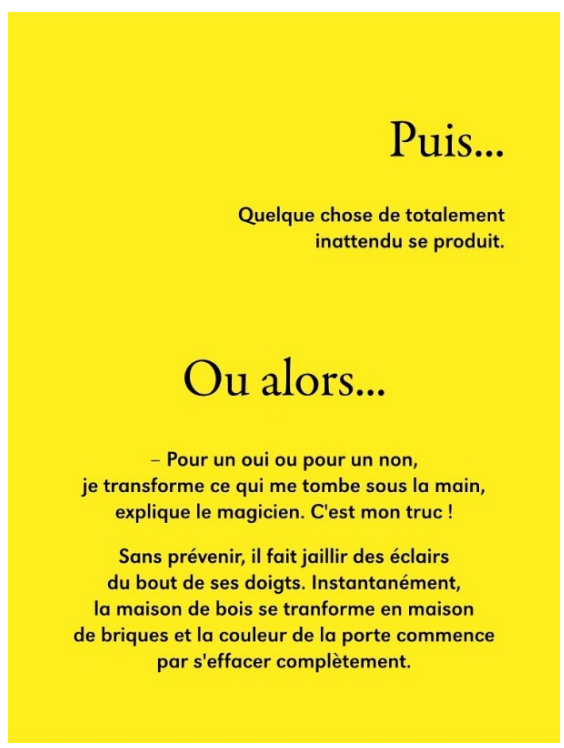

Figure 5. Le lapin bricoleur (E-toiles, 2015).

Dans tous les cas, seulement un nombre réduit de gestes et d'actions est admis.

\section{Résultats}

L'analyse des conduites corporelles et des retours métagestuels des enfants observé·e·s a mené à dégager des aspects de leurs représentations des manipulations narratives programmées et réalisées, que nous proposons de restituer en les reliant, dans un premier temps, à la compréhension et à l'interprétation des œuvres et, dans un deuxième temps, à la perception du rôle des mouvements effectués pour la dynamique de l'œuvre.

\subsection{Gestes et actions comme manifestation de la compréhension et de l'interprétation}

En ce qui concerne la réponse effective des membres de notre échantillon aux requêtes d'interaction des applications, les données indiquent que les gestes et les actions réalisés à l'écran sont pour la plupart significatifs, cohérents avec le récit et, autant que possible, proches ou inspirés des mouvements respectifs dans le monde réel.

Dans le cas de Moi, j'attends..., par exemple, nous avons observé que les enfants adaptent leurs gestes d'une scène à une autre et qu'il·elle·s optent souvent pour des actions ayant une ressemblance avec leurs référents dans le monde empirique : il·elle·s choisissent, par exemple, de tirer verticalement le fil vers le bras du protagoniste lorsqu'il·elle·s reconnaissent le tube d'une perfusion; il·elle's le coupent diagonalement quand il·elle's identifient l'image d'un cordon ombilical et ont tendance à le caresser gentiment quand il représente un démêlé entre époux. Ces différences de mise en œuvre gestuelle semblent indiquer que les jeunes interlecteur rice's comprennent ce qui est en jeu dans chaque scène, mais aussi qu'il·elle·s acceptent d'actualiser leurs interprétations en se conformant au parcours voulu par l'œuvre.

Cette approche consciente de l'interactivité est également visible à travers les difficultés de manipulation observées.

L'une des interlectrices d'Avec quelques briques, par exemple, n'a pas saisi l'image de l'inondation de larmes qu'elle était censée provoquer en inclinant le dispositif. Elle est restée immobile devant l'écran pendant plus de deux minutes et a dû relire certains passages de l'album avant d'être prête à interagir. Au cours de l'entretien, elle est revenue sur ses difficultés, en expliquant qu'elle n'était pas sûre de sa compréhension et, par conséquent, des gestes attendus : «Je savais que le petit garçon avait une espèce de problème, mais je ne savais pas comment faire, comment le déplacer (...) Après j'ai compris, que je devais le faire pleurer car son cœur est comme prisonnier, il n'est pas content. (...) Il est seul et triste, mais au début je ne comprenais pas, puis j'ai compris comment l'aider. J'ai tourné l'iPad et fait sortir les larmes, il y a eu une inondation et la tristesse est partie ».

$\mathrm{Si}$, pour cette participante, l'interaction est clairement inhibée par un problème de compréhension, les données suggèrent, de manière plus générale, une corrélation entre la compréhension et l'interprétation de l'histoire et la manipulation de l'interface narrative.

L'analyse des données a en effet révélé que des mouvements plus ou moins cohérents correspondent à une appréhension plus ou moins riche des histoires, soit à des retours plus ou moins complets en termes de rappel de récit, d'identification des personnages ou d'analyse de la portée symbolique de quelques passages ou éléments narratifs. 
Ainsi, par exemple, le parcours de lecture de Moi, j'attends... du Lecteur 1 a été caractérisé par de nombreuses hésitations et par une mise en œuvre gestuelle non systématiquement conforme aux actions attendues. Le mouvement choisi pour «couper» le cordon ombilical, par exemple, ne semble pas relié à la sphère sémantique de la section : l'enfant n'a pas opté pour un geste diagonal ou net, mais a au contraire répété ce glissement vers la droite qu'il avait appris à automatiser lors des interactions précédentes. De même, au moment de dissiper les incompréhensions du couple, représentées par un nœud qui tient les deux personnages distants, il se montre hésitant, ses manipulations n'étant entamées qu'après la sollicitation visuelle de l'application et consistant à essayer de trainer le fil vers la droite. Son retour sur l'œuvre, très succinct, s'est révélé une simple synthèse de quelques moments significatifs de l'histoire. En parallèle, l'articulation des mouvements au récit semble faible et, en général, ses retours métagestuels ne montrent pas d'association systématique entre les actions réalisées et les passages concernés. Interrogé sur ses mouvements, le sujet se limite à rappeler que le fil était « toujours à donner à la personne » et, même après avoir été invité à réfléchir de manière plus poussée aux gestes effectués lors de passages spécifiques - par exemple au moment de décrire les scènes de l'accouchement ou de la dispute entre les amoureux -, il ne parvient pas à dépasser la simple description des aspects techniques de son mouvement et se limite à illustrer le geste réalisé (« tu devais toujours faire comme ça [en glissant son doigt sur l'écran de gauche à droite] (...) parce que sinon ça faisait rien ») ${ }^{9}$.

Si ces exemples montrent des difficultés, de manipulation et de compréhension, les parcours d'autres sujets révèlent en même temps une manipulation de l'écran cohérente avec les mouvements attendus dans les différents passages, une bonne compréhension de l'histoire, des tentatives d'interprétation sur le contexte des évènements et sur la complexité des attentes du personnage.

Tel est le cas de la Lectrice 2, lors de sa lecture de Moi, j'attends.... Ses actions et gestes se sont révélés cohérents avec ceux présupposés dans les différents passages ${ }^{10}$ et, dans l'entretien, elle s'est montrée capable de revenir à la fois sur les vicissitudes du personnage et sur les différentes fonctions du fil, qu'elle a présentées en articulant ses choix haptiques à ses hypothèses interprétatives : elle déclare qu'elle «a mis» le fil sur l'un des personnages, qu'elle l'a «défait», « coupé », voire " démêlé » pour apaiser l'atmosphère entre les époux. Elle a ajouté que ses choix gestuels ont été systématiquement orientés par les illustrations («J'ai fait ce que le dessin me demandait») qui, à son avis, appelaient à des actions précises : l'image de l'accouchement réclamait de manière sans équivoque de "couper» le cordon ombilical et celle de la dispute de " démêler» les incompréhensions. Les variations lexicales utilisées pour commenter les choix méta-gestuels effectués indiquent que cette lectrice, comme d'autres, a bien perçu la diversité de ses actions et qu'elle a consciemment adapté ses mouvements aux situations narratives.

\subsection{Gestes et actions comme déclencheurs de la dynamique de l'œuvre}

De manière analogue, les participant·e·s semblent avoir globalement perçu la nécessité d'interagir avec l'écran pour dévoiler les contenus. Cinq sujets ont ouvertement associé leur démarche haptique à un mode d'accès à l'histoire, voire au fait de parcourir le texte en le feuilletant. Deux ont plus précisément reconnu leurs manipulations comme des évènements programmés pour actualiser le texte et pour découvrir l'histoire.

Chez la plupart des interlecteur·rice's de Moi, j'attends..., l'interaction avec le fil est clairement identifiée comme la manière de déclencher l'avancement narratif: ainsi, les enfants considèrent-il·elle's que leurs gestes et actions permettent à l'histoire de "continuer» (Lecteur 6) ou de se «débloquer » (Lectrice 7), voire aux "pages » et aux « images » de se déployer (Lecteur 4). Pour le Lecteur 1, non seulement l'intervention sert à contrer l'inertie de la scène (« parce que sinon ça fait rien »), mais également à épauler le personnage, en agissant pour lui quand « il restait comme ça, bloqué ».

En ce qui concerne les interlecteur·rice's de Mon voisin, tou·te·s les participant·e·s ont été capables de revenir sur les modes de circulation adoptés, avec un degré plus ou moins fin de précision descriptive, mais seulement cinq (Lectrices 2,3 et 9 et Lecteurs 5 et 8 ) ont ouvertement associé leur démarche haptique à un mode d'accès à l'histoire. La Lectrice 2, par exemple, a été en mesure de revenir aussi bien sur son processus d'exploration de l'espace fictionnel que sur la nécessité d'intervenir sur l'écran pour accéder à l'histoire. Lors de l'entretien, elle a mentionné l'utilisation des flèches et la possibilité de «toucher sur un mur» pour se glisser dans l'appartement du voisin, puis a expliqué que son exploration de l'immeuble était nécessaire pour «connaitre l'histoire». De même, le Lecteur 5 a pu relier son déplacement dans le bâtiment à la découverte de l'histoire et des lieux du récit (« Ici, il y avait une espèce de tour, puis ça se voyait, que c'était haut, avec beaucoup d'étages (...) et bah, je devais monter et descendre les étages et regarder et

\footnotetext{
${ }^{9}$ Malgré ses difficultés de compréhension, le Lecteur 1 démontre avoir perçu la contrainte posée par son intervention sur l'avancement du récit. Interprétée comme fonctionnelle au dénouement des vicissitudes du personnage, l'interaction est à la fois décrite comme une condition nécessaire à la progression de l'œuvre et comme une aide permettant au héros de sortir d'une situation sans issue : selon les mots de cet enfant, son intervention sert notamment «à faire repartir le monsieur » et permet de «l'aider pour s'en sortir ».

${ }^{10}$ Par exemple, au moment de la première rencontre entre le personnage et son amoureuse, la lectrice a correctement répondu à la requête d'une triple interaction avec l'écran, en répétant trois fois le mouvement de tirer et accompagner le fil à travers l'écran ; au moment de couper le cordon ombilical, elle a choisi un mouvement ample et long, avec lequel elle a traversé diagonalement le brin rouge ; dans la scène prévoyant de détendre l'atmosphère entre les amoureux en étirant le fill, elle a opté pour un mouvement large, léger et déployé sur la longueur de la tablette.
} 
ça servait à découvrir un peu l'histoire et un peu la maison »). Selon les Lectrices 3 et 9, ainsi que selon le Lecteur 8 , les mouvements d'exploration des plans vertical et horizontal programmés par l'application étaient même assimilables à une opération de feuilletage, permettant de «lire » ou de « voir » l'histoire. Ainsi, après avoir détaillé son parcours, la Lectrice 3 a identifié ses gestes de navigation (monter et descendre les étages du bâtiment, glisser dans l'appartement du voisin) comme des modes d'accèsà l'œuvre, qu'elle a comparés à une manière de tourner des pages quand on n'en a «pas vraiment ». Pareillement, le Lecteur 8 a affirmé avoir feuilleté des pages aussi bien en montant d'étage en étage par le biais des flèches qu'en explorant le plan horizontal à partir de la cloison, ces deux mouvements étant considérés comme également indispensables "pour voir l'histoire». Plus vague, mais également explicite, la réponse de la Lectrice 9, pour laquelle les mouvements effectués étaient « quelque chose » de nécessaire " pour lire ».

Ces différents propos suggèrent que les enfants de notre échantillon ont une perception claire de leur propre rôle dans la configuration de l'œuvre.

Si quelques sujets - généralement ceux·celles qui ont montré des difficultés de compréhension et manipulation considèrnt qu'il·elle·s ont simplement obtempéré aux requêtes de l'application, d'autres perçoivent clairement la possibilité de co-construire l'histoire et la tentative de l'œuvre littéraire numérique de les embarquer dans un environnement immersif. Dans ce sens, il·elle·s comprennent que l'application les met dans la condition de vivre les aventures des personnages, d'en ressentir les émotions comme s 'il·elle·s étaient à leur place («c'était comme si j'étais lui ») et d'en déterminer les comportements (« quand je monte les escaliers, il (= le personnage) monte à l'étage, quand je tourne à droite, il vient avec moi»). En d'autres termes, les membres de notre échantillon sont globalement conscients de la fonction de leurs gestes et actions pour la dynamique de l'œuvre, ainsi que des effets de leurs interventions sur le récit.

\section{Conclusion}

Notre observation a montré que les jeunes interlecteur·rice's suivi·e·s dans le cadre notre recherche acceptent d'intégrer l'espace fictionnel pour construire le sens, en intervenant sur l'interface narrative comme les œuvres littéraires l'ont établi (du moins, si aucun problène de compréhension ne freine ou n'inhibe leurs mouvements). Leur action peut être considérée comme le résultat d'un choix délibéré et significatif, qui correspond à la fois aux intentions du texte et à une disponibilité subjective à s'impliquer dans l'espace fictionnel. Dans cette perspective, il semblerait, en accord avec notre hypothèse de départ, que les interlecteur rice's se trouvent dans une position double, qui les veut à la fois en tant qu'individus singuliers, qui reçoivent l'œuvre selon leur sensibilité, leur connaissance et leur vécu, et en tant qu'alterego empiriques d'un modèd programmé par l'œuvre. Les différences de mise en œuvre observées, tout comme l'analyse des conduites verbales, témoignent, en effet, de parcours contraints et, en même temps, d'attitudes ou de manièresde construire le sens personnelles et diverses d'un sujet à l'autre. Les interventions des sujets sur écran traduisent leurs points de vue sur le contexte narratif, mais également sur le monde réel, dont il·elle·s sont amené·e·s à reconnaitre les analogies avec les éléments exploités à l'écran. Elles témoignent également de formes diverses d'acceptation de ce glissement entre un rôle lectoral externe, commun à tout $\cdot e$ lecteur $\cdot$ rice, et un rôle interne, propre à un·e interlecteur·rice amené·e à intervenir « de manière non triviale » (Aarseth, 1997) sur le texte. Paradoxalement, ni le plaisir de la manipulation de l'écran, déclaré par tou·te·s les participant·e·s, ni des compétences technologiques plus poussées n'ont affecté les résultats. Au contraire, les sujets ayant déclaré avoir des compétences préalables ou bien ayant fait référence à leurs pratiques de lecture et de jeux en ligne ou sur d'autres dispositifs numériques, ne se sont pas avérés favorisé·e $\cdot s$, en étant au contraire par moments entravé $\cdot \cdot \cdot s$ par la tendance à vouloir reproduire des gestes et des actions familì̀es ou expérimentées en d'autres contextes. Dans cette expérience, tout comme dans celles qui ont suivi (Acerra, $2019 ; 2020)$, se sont avéré·e·s favorisé·e·s sur les plans de la compréhension et de l'interprétation les élèves capables de reconnaitre la dimension gestualisée de la lecture, et donc de regarder l'interaction comme une manifestation d'un parcours de lecture, ancrée au récit et signifiante.

\section{Références}

Aarseth Espen. (1997). Cybertext. Perspectives on ergodic literature. Baltimore : Johns Hopkins University Press.

Acerra Eleonora. (2019). Les applications littéraires pour la jeunesse: ouvres et lecteurs. Thèse en Littérature française, l'Université Paul Valéry - Montpellier 3.

Acerra Eleonora. (2020). Lire les lecteurs numériques : d'une synthèsedes recherches à une expérience de lecture d'application littéraire en classe de CM1-CM2. Revue de recherches en littératie médiatique multimodale, $\mathrm{n}^{\circ} 12$, Université du Québec à Montréal. [URL : https://litmedmod.ca/sites/default/files/pdf/r2-lmm_vol12_acerra.pdf]

Bandura Albert. (2006). Toward a psychology of human agency. Perspectives on Psychological Science, $\mathrm{n}^{\circ} 1$, p. 164180.

Bélisle Claire. (2011). Les nouveaux environnements de lecture. In C. Bélisle (éds.), Lire dans un monde numérique. Villeurbanne : Presses de l'Enssib - Collection Papiers, p. 180-204.

Bezemer Jeff et Kress Gunther. (2015). Multimodality, learning and communication. A social semiotic frame. Oxon et New York : Routledge. 
Bouchardon Serge. (2008a). La littérature numérique. Terminal [En ligne], 101 | 2008. [URL: http://journals.openedition.org/terminal/4472].

Bouchardon Serge. (2008b). Une esthétique de la matérialité. In A. Saemmer et M. Maza (éds.), E-formes. Écritures visuelles sur supports numériques. Saint-Étienne : Publications de l'Université de Saint-Étienne, p. 135-144.

Bouchardon Serge. (2009). Littérature numérique : Le récit interactif. Paris : Hermès.

Bouchardon Serge (2010). Déprise. [Dernière mise à jour: 2019]. [URL: https://apps.apple.com/ca/app/déprise/id1434229807?l=fr]

Bouchardon Serge. (2011). Des figures de manipulation dans la création numérique. Protée. Esthétiques numériques. Textes, structures, figures, vol. $39 \mathrm{n}^{\circ} 1$, p. 37-46.

Bouchardon Serge. (2014). La valeur heuristique de la littérature numérique. Paris : Hermann.

Carré Philippe. (2003). La double dimension de l'apprentissage autodirigé, Contribution à une théorie du sujet social apprenant. Revue Canadienne pour l'étude de l'éducation des adultes [URL: http://journals.msvu.ca/index.php/cjsae/article/view/1871/1632]

Demers Stéphanie, Bachand Charles-Antoine et Leblanc Claudia. (2016). Les approches inductives au service de l'agentivité épistémique et des finalités éducatives émancipatrices. Approches inductives, $\mathrm{n}^{\circ} 3(2)$, p. 41-70. [URL : https://doi.org/10.7202/1037913ar].

Dorléans Marie (2013). Mon voisin. Paris: Éditions des Braques et Tralalere. [URL : https://books.apple.com/fr/book/mon-voisin/id745441239]

Eco Umberto. (1985). Lector in fabula ou La coopération interprétative dans les textes narratifs. Paris : Grasset.

e-Toiles. (2015). Le lapin bricoleur. [URL : https://books.apple.com/fr/book/le-lapin-bricoleur/id1063318741]

France Télévisions. (2013). Moi, j'attends... [Dernière mise à jour : 2017]. [URL : https://itunes.apple.com/fr/app/moijattends/id733823646? $\mathrm{mt}=8$ ]

Jeanneret Yves. (2000). Y-a-t-il (vraiment) des technologies de l'information? Nouvelle édition revue et corrigée. Lille : Presses du septentrion.

Jeanneret Yves et Souchier Emmanuel. (1999). Pour une poétique de l'écrit d'écran. Xoana, nº 6, p. 97-107.

Johnson Mark. (2007). Meaning of the body: Aesthetics of human understanding. Chicago, Illinois: The University of Chicago Press.

Murray Janet. (1997). Hamlet on the Holodeck. The Future of Narrative in Cyberspace. Cambridge : MIT Press.

Pelard Emmanuelle. (2018). Poésies numériques tactiles: toucher les signes par la «main de l'œil», manipuler la matière à l'écran. Itinéraires, 2017-3. [URL : http://journals.openedition.org/itineraires/3983].

Saemmer Alexandra. (2007). Matières textuelles sur support numérique. Saint-Etienne : Publications de 1'Université de Saint-Etienne.

Saemmer Alexandra. (2008). Figures de style électroniques. In M. Maza et A. Saemmer (éds.), E-formes. Écritures visuelles sur support numérique. Saint-Etienne : Publications de 1'Université de Saint-Etienne, p. 145-157.

Saemmer Alexandra. (2011). Hypertexte et irradiation iconique. Les cahiers du numérique, vol. 7, n 3-4, p. 47-69. [URL : https://www.cairn.info/revue-les-cahiers-du-numerique-2011-3-page-47.htm]

Saemmer Alexandra. (2015). Rhétorique du texte numérique. Figures de la lecture, anticipations de pratiques. Villeurbanne : Presses de l'Enssib.

Saemmer Alexandra et Tréhondart Nolwenn. (2014). Les figures du livre numérique augmenté au prisme d'une rhétorique de la réception. Études de communication - Langages, information, médiations, n 43, p. 107-128.

Weissberg Jean-Louis. (1999). Retour sur interactivité. Revue des sciences de l'éducation, n 25(1), p. 167-199. [URL : https://doi.org/10.7202/031997ar.] 